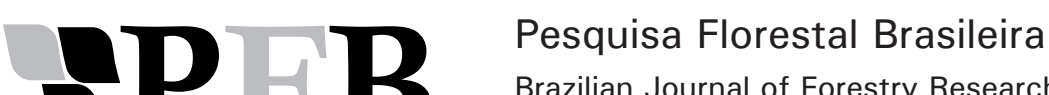 \\ Brazilian Journal of Forestry Research \\ http://pfb.cnpf.embrapa.br/pfb/
}

\section{Compostos químicos em teca}

\author{
Fernanda Viana da Silva Leonardo, Henrique Figueiredo Rocha ${ }^{1}$, Zaira Morais dos Santos Hurtado de Mendoza ${ }^{*}$ \\ ${ }^{1}$ Universidade Federal de Mato Grosso, Departamento de Engenharia Florestal, Av. Fernando Corrêa da Costa, n² 2367, CEP 78060-900, Cuiabá, MT, Brasil
}

\begin{abstract}
zaira@ufmt.br
Produtos florestais

Madeira

Tecnologia

Index terms:

Forest products

Wood

Technology
\end{abstract}

"Autor correspondente:

Termos para indexação:

\section{Histórico do artigo:}

Recebido em 05 nov 2014

Aprovado em 02 set 2015

Publicado em 30 set 2015
Resumo - Os compostos quinônicos, em sua grande parte, são gerados na fração extrativos das madeiras, em um sistema biológico complexo e variável. Por não fazerem parte da parede celular, eles recebem o nome de compostos secundários. Na literatura tem indicações de uso em diversos segmentos, que vão desde a indústria alimentícia até a indústria farmacêutica. Dentro da área de utilização industrial da madeira, eles são pouco desejáveis, visto que são tratados apenas como substâncias acidentais nas cadeias produtoras de celulose, papel, carvão vegetal e serraria. Apesar de sua pouca quantidade em relação aos outros compostos químicos chamados de fundamentais, essas substâncias vem recebendo atenção especial dos pesquisadores, revelando uma gama diversificada de ofertas para o mercado de produtos têxteis, fármacos, corantes, polímeros e outros compostos. As quinonas são encontradas em fungos, liquens e, em sua grande maioria, nos vegetais superiores. A Tectona grandis, vulgarmente conhecida como teca, é capaz de biossintetizar antraquinonas, composto quinônico, que the confere resistência natural. Esta espécie fornece madeira bastante valorizada no setor moveleiro, podendo também ser explorado os seus metabólitos, tanto para suprir o mercado em compostos quinônicos de uso comercial como para desenvolvimento de novas tecnologias, agregando valor aos plantios dessa espécie dentro do nosso País.

\section{Chemical compounds in teak}

Abstract - Quinone compounds are largely generated at extractive fraction of the woods in a complex and variable biological system. The literature has indications for many segments from food industry to pharmaceutical industry. Within the field of industrial use of wood, they are less desirable since they are treated only as incidental substances in production strings of pulp, paper, charcoal, and sawmill. In spite of its small amount, compared to other chemical compounds called essential, these substances have received special attention from researchers revealing a diverse range of offerings to market products textiles, pharmaceuticals, colorants, and other polymers, for which are being tested and employed. Quinones are found in fungi, lichens, and mostly in higher plants. Tectona grandis, usually called teak, is able to biosynthesize anthraquinones, which is a quinone compound, byproduct of secondary metabolism. This species provides wood that is much prized in the furniture sector and can also be exploited for metabolites to supply the market in quinone compounds and commercial development of new technologies, adding value to the plantations of this species within our country. 


\section{Introdução}

O Brasil e o mundo têm dedicado consideráveis esforços para abertura de novas fronteiras tecnológicas e econômicas, seja para o estabelecimento de matrizes energéticas renováveis, seja para o estabelecimento de biorrefinarias que dinamizem a conversão de biomassa em biocombustíveis, insumos químicos, materiais, alimentos, rações e/ou energia, com otimização de uso, minimização de resíduos e maximização de lucros.

Os produtos químicos desenvolvidos a partir de coprodutos e resíduos, são os que possuem maior potencial de agregação de valor às cadeias produtivas de biomassa. A indústria química tem participação estratégica dentro das linhas de produção, fornecendo insumos e produtos finais a diversos setores da economia, como o petroquímico, farmacêutico, automotivo, construção, agronegócio, cosméticos, dentre outros (Embrapa Agroenergia, 2011).

Os compostos quinônicos, também conhecidos como quinonas, são compostos químicos amplamente conhecidos no mundo da química, e no caso especial dos vegetais superiores, eles estão concentrados na fração da madeira denominada extrativos, sendo sua síntese realizada por um sistema biológico complexo e variável. Eles permeiam a literatura com aplicações e implicações em diversos segmentos, porém, pouco desejáveis quando tratados apenas como substâncias acidentais nas grandes áreas de uso da madeira, como as cadeias produtoras de celulose, papel, carvão vegetal e setor madeireiro. Por outro lado, essas substâncias têm recebido especial atenção de pesquisadores, revelando uma gama diversificada de oferta para o mercado de produtos têxteis, fármacos, corantes, polímeros e outros, sendo isoladas, testadas e empregadas com sucesso (Souza, 2007).

As quinonas dos vegetais superiores podem ser extraídas das madeiras, utilizando-se solventes orgânicos voláteis, soluções alcalinas diluídas ou água quente, podendo também ser isolada por destilação em corrente de vapor de água. De acordo com Gibaja (1998), o correto isolamento e utilização destes compostos poderia ampliar o rol de insumos químicos naturais, atendendo assim uma demanda cada vez mais crescente do mercado consumidor.

Este trabalho teve como objetivo fazer um levantamento bibliográfico sobre os principais compostos químicos presentes na madeira de teca (Tectona grandis), visando agregar valor a essa espécie.

Pesq. flor. bras., Colombo, v. 35, n. 83, p. 315-322, jul./set. 2015

\section{Composição química da teca}

A teca (Tectona grandis) é uma espécie arbórea latifoliada da família Lamiaceae (ex Verbenaceae), popularmente conhecida como teca. É nativa do subcontinente índico e sudeste asiático, dotada de alta adaptabilidade, podendo ser cultivada em diferentes regiões tropicais (Chaves \& Fonseca, 1991; Angeli, 2003).

A combinação de beleza, resistência e durabilidade fez da madeira desta espécie uma das mais valiosas do mundo, superando outras madeiras nobres, como a de mogno - Swietenia macrophylla King (Tsukamoto Filho et al., 2003). Seu cultivo, que era então limitado à região da Ásia Tropical, passou a ocupar outras zonas tropicais, como África Ocidental, América Central e América do Sul, com expressiva densidade de indivíduos no Brasil e Costa Rica (Figueiredo et al., 2005).

No Brasil, os plantios de teca iniciaram-se no final da década de 1960, implantados pela empresa Cáceres Florestal, na região de Cáceres, MT. As condições edafoclimáticas adequadas, aliadas às boas práticas silviculturais, contribuíram para a redução do seu ciclo de produção de 80 para 25 anos (Tsukamoto Filho et al., 2003).

De acordo com Angeli (2003), a durabilidade é uma característica marcante dessa espécie e que até o momento são poucos os registros, nos países onde a teca é cultivada, de ataques de pragas que possam comprometer os plantios. A autora relata que a resistência natural do cerne da teca deve-se a tectoquinona, um preservativo natural contido nas células da madeira. Moreira et al. (2006) corrobora com essa afirmativa, relatando que a tectoquinona, é um composto químico pertencente à classe das antraquinonas, e que vários estudos a indicam como responsável pela proteção natural dessa madeira. Motta et al. (2013) caracterizaram a madeira de teca como imune à ação dos fungos apodrecedores e insetos, podendo ser enterrada, exposta ao tempo ou à água do mar sem sofrer grandes danos, elencando-a também entre as espécies preferidas para o reflorestamento, para a produção de madeira sólida e laminados.

Entretanto, Oliveira et al. (2012) ressaltam que nenhuma espécie de madeira, nem mesmo aquelas de reconhecida durabilidade natural, é capaz de resistir, indefinidamente, às intempéries, variações das condições ambientais, ataque de microrganismos e à ação do próprio homem. 
Floréz (2012), ao caracterizar quimicamente a madeira de teca, obteve valores médios de cinzas, extrativos totais, lignina e holocelulose, respectivamente iguais a $0,70 \%, 8,94 \%, 32,47 \%$ e $57,94 \%$, em árvores amostradas em plantio experimental com 13 anos de idade, estabelecido no noroeste do estado de Minas Gerais. Contudo, o autor não fez a análise química qualitativa desses compostos.

Resultados semelhantes ao de Floréz (2012) referente aos teores totais de extrativos em teca foram obtidos por Guerra (2010) em madeiras provenientes do município de Jerônimo Monteiro, ES, nas posições de cerne interno, intermediário, externo e de alburno $(8,87 \% ; 8,80 \%$; $9,94 \%$ e $5,31 \%$, respectivamente). Este mesmo autor, ao avaliar a resistência natural das madeiras de Eucalyptus cloeziana, Corymbia torelliana, Casuarina sp., Acacia mangium e teca, constatou que em se tratando do ataque de cupins xilófagos, apenas a madeira de teca apresentou relação direta entre a resistência a esses microorganismos e o teor de extrativos.

Paes et al. (2007), ao avaliarem a resistência natural das madeiras de leucena (Leucaena leucocephala), louro pardo (Cordia trichotoma), jurema-preta (Mimosa tenuiflora), marmeleiro preto (Croton sonderianus), sabiá (Mimosa caesalpiniifolia), nim indiano (Azadirachta indica) e teca aos fungos Postia placenta e Polyporus fumosus e ao térmita Nasutitermes corniger, verificaram que o cerne externo (região fronteiriça com o alburno, formada pela planta mais madura) da madeira de teca foi moderadamente resistente.

Oliveira Junior et al. (2012) testaram a hipótese que se atribui resistência ao ataque de fungos xilófagos à presença de tectoquinona, avaliando o efeito do extrato hidroalcoólico de teca em diferentes condições de termotratamento e in natura sobre o fungo Agrocybes cf. perfecta. Os resultados obtidos negaram a hipótese inicial para a espécie de xilófago considerada, pois o extrato hidroalcoólico não apresentou ação inibitória ou resistência ao desenvolvimento do referido fungo.

Sobre a tectoquinona extraída da madeira de teca, ainda não existem relatos da sua possível ação fungicida e inibitória ou resistência natural, sobre as espécies de fungos patógenos Cylindrocladium parasiticum, Olivea tectonea e Ceratocystis fimbriata, cujas ocorrências oficiais foram em 2008 e 2009 no Brasil (Bonaldo et al., 2011; Poltronieri et al., 2011; Firmino et al., 2012). Com relação ao patógeno Ceratocystis fimbriata, causador da doença vascular em teca, não há, até o momento, informações precisas sobre sua dispersão, sendo difícil correlacionar a presença ou ausência desse fungo, com a durabilidade natural da espécie.

Fengel \& Wegener (1989) mencionam que a resistência natural das madeiras, ao ataque de insetos e fungos, está intimamente relacionada com a presença de substâncias do metabolismo secundário das plantas, conhecidas como extrativos. Sarto \& Sansigolo (2010) assim como Pinto (2007), ressaltam que a correlação entre esses dois fatores nem sempre pode ser tomada como regra, visto que isso dependerá do tipo e quantidade do composto químico extraído da madeira, além dos tipos de fungos e térmitas que as estariam atacando.

Klock et al. (2005) enfatizam que os compostos secundários são conhecidos como extrativos e tem como principal característica a baixa massa molecular. Essas substâncias são muitas vezes responsáveis pelas características organolépticas e, embora tenha pequena expressão na massa total da madeira, elas influenciam notadamente nas propriedades e no processamento da mesma. As espécies de madeira variam grandemente quanto à resistência à degradação, embora somente o cerne da madeira apresente resistência significativa. Esta resistência é atribuída aos compostos químicos, frequentemente fenólicos, que são encontrados na fração de extrativos da madeira, e que são tóxicos a fungos e insetos.

Dentre os metabólitos secundários das plantas, as substâncias fenólicas são classificadas em flavonóides, ácidos hidroxicinâmicos e derivados, fenóis simples e também em ácidos fenólicos, que neste caso têm como representantes os compostos quinônicos ou simplesmente quinonas (García \& Daoud, 2002).

As quinonas constituem uma classe especial de dicetonas cíclicas $\alpha$ e $\beta$ não saturadas. As antraquinonas são, contudo, o grupo mais amplo das quinonas naturais e são encontradas em fungos, liquens e vegetais superiores. Nas plantas superiores localizamse predominantemente no xilema, córtex e raízes. As quinonas do tipo fitoquinonas, naftoquinonas, benzoquinonas, ubiquinonas e plastoquinonas estão também amplamente distribuídas em diferentes famílias de vegetais superiores, e servem inclusive para classificálos botanicamente (González et al., 1971).

De acordo com Gibaja (1988), a característica marcante dos pigmentos quinônicos é a sua cor e esta coloração varia do amarelo, passando por alaranjado e vermelho, 
até o preto. Em se tratando de grupamento químico, eles podem ser agrupados em benzoquinonas, naftoquinonas, antraquinonas e quinonas policondensadas. Quanto ao sistema cíclico, elas geralmente são classificadas em benzoquinonas, naftoquinonas, antraquinonas $\mathrm{e}$ fenantraquinonas, tendo respectivamente, como sistema aromático, anel benzênico, naftalênico, antracênico e o anel fenantrênico (Sousa, 2012).

De um modo geral, as quinonas naturais mais representativas são de vital importância para os vegetais superiores, artrópodes, fungos, liquens, bactérias, algas e vírus. A distribuição dessas substâncias nos diferentes organismos implica, possivelmente, em funções biológicas múltiplas, agindo de forma conspícua em seus diversos ciclos bioquímicos (Silva et al., 2003).

González et al. (1971) observaram que todas as antraquinonas, obtidas em espécies das famílias Verbenaceae, Rubiaceae e Bignoniaceae, são do tipo simples, predominando as hidroxiladas e as metoxiladas, excetuando-se a tectoquinona e seus subprodutos de oxidação, que são comuns nas três famílias.

As quinonas são encontradas também no ar atmosférico, podendo ocorrer nas fases vapor, líquida ou particulada. São emitidas diretamente a partir de combustão incompleta de matéria orgânica, especialmente combustíveis fósseis, sendo esta a principal fonte antrópica direta de emissão de quinonas (Sousa, 2012).

Moreira et al. (2006), através de procedimentos fitoquímicos clássicos, com ênfase para o isolamento da tectoquinona a partir de extrato hexânico de caule, atestaram que espécimes de reflorestamento de teca, com 10 anos de idade, são capazes de biossintetizar a antraquinona, que é a responsável pela durabilidade dessa madeira. Além da tectoquinona, outras três substâncias também foram isoladas pelos mesmos autores: lapachol, obtusifolina e desidro- $\alpha$-lapachona.

O lapachol pode ser obtido por vários métodos de extração, sempre se baseando na sua solubilidade em solvente orgânico e a do seu sal em meio aquoso alcalino, gerando soluções amarelas e vermelhas, respectivamente (Fonseca et al., 2003).

Nidavani \& Mahalakshmi (2014) relataram em sua revisão, que para a madeira de teca, a maior concentração de lapachol e tectocquinona encontra-se nas folhas e no cerne, e que esses compostos, juntamente com outros alcaloides, conferem durabilidade natural a essa madeira.

\section{Usos dos compostos químicos da teca}

Souza et al. (2007), em seus trabalhos com Tectona grandis, verificaram que a espécie tem potencial para o desenvolvimento de tecnologias a partir do aproveitamento de seus metabólitos, como por exemplo, para produção de corante natural empregado na indústria de tintas e nas artes plásticas. A partir da extração hidroalcoólica da parte aérea dessa planta, os autores isolaram seis concentrados com grande potencial de utilização como corante natural, porém não foram encontrados traços de tectograndone e de lapachol, substâncias químicas de ocorrência comum na espécie. Nidavani \& Mahalakshmi (2014), também indicam a espécie como promissora para produção de corantes naturais e ressaltam que a quantidade de compostos fitoquímicos extraídos depende do método de isolamento e da parte da árvore utilizada.

Mendoza \& Borges (2013) estudando os compostos cromóforos das folhas de teca, para fins de corantes naturais, verificaram via sistema Munsell e CIELAB, que a escala de cores para os extratos aquosos ficaram entre o alaranjado e o vermelho, e que esses compostos predominavam nos verticilos apicais. Os autores ressaltam, também, que devido à espécie ser caducifólia, a taxa de clorofila e de fotossíntese é passível de alteração, o que leva a deduzir que a concentração dos compostos cromóforos, poderá ser afetada pela época do ano em que as folhas são coletadas. Essa hipótese é fortalecida pelos trabalhos de Goergen et al. (2015), que verificando a dinâmica temporal de clorofila nas folhas de Eucalyptus grandis, encontraram diferentes concentrações desse pigmento de acordo com as quatro diferentes estações do ano, sendo o período verãooutono, o de maior conteúdo de clorofila e de máxima produção fotossintética.

Morillo (2009), ao estudar folhas de teca para extração de corantes naturais, constatou que o grupo predominante era o das quinonas, cujos compostos foram identificados como sendo 2-metil-9,10-antraquinona (tectoquinone) 1,5-di-hidroxi-2-metil-9,10-antraquinona e o lapachol ou 2-hidroxi-3-(3-metil-2-butenil)-1,4-naftoquinona. Esses compostos foram testados como corante natural para tingimento de tecidos, revelando-se potencialmente apropriados para esse fim.

De acordo com Bergamini et al. (2005), o uso de antraquinonas é bem aceito no setor de corantes. Os autores mencionam que o Reactive Blue 4-RB4, um dos 
corantes reativos mais estudados, possui em sua estrutura uma antraquinona como grupo cromóforo e um grupo diclorotriazina como grupo reativo. Ele é amplamente utilizado na indústria têxtil, havendo inúmeras pesquisas para melhorar a eficiência de fixação dessa classe de corantes.

No processo de polpação soda, as antraquinonas recebem ainda aplicação tecnológica em substituição ao sulfeto. Fraga et al. (2002) mencionam que a adição de antraquinona ao processo soda promove um aumento dos rendimentos bruto e depurado, resultando em pronunciada melhoria da eficiência de deslignificação. Segundo os autores, o rendimento (49\%) e a qualidade da polpa produzida com as antraquinonas foram semelhantes aos da polpa Kraft obtida com a madeira de Pinus taeda.

Ao avaliarem o efeito da antraquinona no processo de polpação soda e Kraft sobre cavacos pré-hidrolisados de madeira de Eucalyptus grandis, Caraschi et al. ( 2007) concluíram que o uso de antraquinona, proporcionou um pequeno acréscimo no rendimento do teor de celulose em ambos os processos. Eles também verificaram que a polpa obtida pelo processo soda apresentou melhor qualidade quanto ao grau de pureza química do que o processo Kraft, verificado pelo número kappa, álcali residual, teor de sólidos e teor de ácidos hexenurônicos.

Cowan (1999) ressalta que as quinonas podem se tornar substratos indisponíveis para microrganismos, e tal como acontecem com todos os agentes antimicrobianos derivados de plantas, os possíveis efeitos tóxicos das quinonas devem ser cuidadosamente examinados. Segundo Martí (1998), as quinonas tem ação muito irritante, podendo causar fenômenos gerais de intoxicação em forma de mal-estar, náuseas, tonturas e fraquezas nos braços e pernas, dor de garganta, tosses e ataques violentos de espirros. O autor relata que essas substâncias apresentam riscos para os operadores que manuseiam peças e resíduos de madeiras que contenham essa e demais substâncias químicas.

Sousa et al. (2003) descrevem que as antraquinonas possuem efeito laxativo e Silva et al. (2003) relatam que a interferência das quinonas na apoptose (suicídio celular) constitui-se hoje uma pesquisa interdisciplinar de fronteira na química medicinal, existindo grande expectativa quanto à delineação de estratégias racionais visando o combate de neoplasias.

O lapachol e a $\beta$-lapachona podem ser consideradas as naftoquinonas que mais influenciaram os grupos brasileiros de pesquisa em química e farmacologia de quinonas. Apesar de ainda não ser um fármaco, a $\beta$-lapachona é uma substância muito importante do ponto de vista da pesquisa científica (Cruz et al., 2012).

Com motivação encontrada no grande prejuízo que as plantas daninhas causam à agricultura, a capacidade fitotóxica de seis quinonas comerciais (2,5-dihidroxi-1,4-benzoquinona, canforquinona, fenantrequinona, antrarrufina, antraquinona sulfonato de sódio e lapachol) foram testadas por Ferreira et al. (2000) no controle de plantas de Sorghum bicolor, Cucumis sativus, Desmodium tortuosum, Lactuca sativa, Cucumis sativus, Desmodium tortuosum, Hyptis suaveolens e de Euphorbia heterophylla. As quinonas canforquinona, fenantrequinona, antrarrufina e lapachol causaram inibição significativa $(9,52$ a $71,43 \%)$ no sistema radicular de Hyptis suaveolens, mostrando-se potencialmente eficazes para o controle desta erva.

Nidavani \& Mahalakshmi (2014), em seu trabalho de revisão, concluíram que os compostos químicos encontrados na árvore de teca têm inúmeras atividades farmacológicas, mas poucos foram explorados de forma comercial.

\section{Considerações finais}

Apesar dos usos já consagrados da madeira de teca, como produção de peças de usos nobres, movelaria, marcenaria, construção civil e naval, o conhecimento da versatilidade, incluindo a distinção dos componentes químicos, geradores de produtos não madeireiros desta e de outras espécies, permite relacionar múltiplas áreas da ciência para o desenvolvimento e aplicação de tecnologias provenientes de fontes renováveis.

Assim sendo, a utilização de insumos químicos, como os quinônicos, despontam como uma alternativa importante para as áreas de preservação natural, corantes naturais, farmacologia e cosmetologia. A utilização desses compostos naturais corrobora para a implementação de um novo conceito de produção e desenvolvimento, com vistas a integralizar o aproveitamento dos recursos florestais e a consequente redução de impactos ambientais.

\section{Referências}

ANGELI, A. Tectona grandis (Teca). In: IDENTIFICAÇÃO DE ESPÉCIES FLORESTAIS. Piracicaba: IPEF, 2003. Disponível em: $<$ http://www.ipef.br/identificacao/tectona. grandis.asp $>$. Acesso em: 24 jun. 2014. 
BERGAMINI, M. F.; OLIVEIRA, F. C. M.; ZANONI, M. V. B. Análise voltamétrica do corante têxtil do tipo antraquinona empregando eletrodos de carbono impresso. Eclética Química, Araraquara, v. 30, n. 2, p. 53-59, 2005. DOI: 10.1590/S010046702005000200007

BONALDO, S. M.; BARCELI, A. C.; TRENTO, R. A.; GASPAROTTO, F.; TAFFAREL, C. Relato oficial da ocorrência de Olivea tectonea em teca (Tectona grandis) no Brasil. Summa Phytopathologica, Botucatu, v. 37, n. 3, p. 153, 2011. DOI: 10.1590/ S0100-54052011000300015

CARASCHI, J. C.; ROSA, R. R.; SANTIAGO, L. F. F.; VENTORIM, G. O efeito da antraquinona no processo de polpação soda e Kraft de Eucalyptus grandis. In: COLÓQUIO INTERNACIONAL SOBRE CELULOSE DE EUCALIPTO, 3., 2007, Belo Horizonte: UFV, 2007. 7 p.

CHAVES, E.; FONSECA, W. Teca: Tectona grandis L. f. especie de Arbol de uso multiple em America Central. Turrialba: CATIE, 1991. $60 \mathrm{p}$.

COWAN, M. M. Plant products as antimicrobial agents. Clinical Microbiology Reviews, Washington, US, v. 12, n. 4, p. 564-58, 1999.

CRUZ, N. A.; ANJOS, A.; SIMÕES, V. N.; BROTTO, D. F.; OLIVEIRA, T. D. Extração e conversão química de uma quinona natural e aplicação do produto em química de coordenação. In: ENCONTRO DE INICIAÇÃO CIENTÍFICA, 10., 2012, Campo Grande. Anais... Campo Grande: UEMS, 2012. p. 1-6.

EMBRAPA AGROENERGIA. Biorrefinarias. 2011. Folder. Disponível em: <http://www.infoteca. cnptia.embrapa.br/handle/ doc/908142>. Acesso em: 24 jul. 2014.

FENGEL, D.; WEGENER, G. Wood: chemistry, ultrastructure, reactions. Berlim:Walter de Gruyter Incorporated, 1989. 613p.

FERREIRA, M. L.; BARBOSA, L. C. A.; DEMUNER, A. J.; SILVA, A. A.; PEREIRA, R. C. Avaliação da atividade herbicida de algumas quinonas. Acta Scientiarum, Maringá, v. 22, n. 4, p. 999-1003, 2000. DOI: 10.4025 /actasciagron.v22i0.2852

FIGUEIREDO, E. O; OLIVEIRA, L. C de.; BARBOSA, L. K. F. Teca (Tectona grandis L.f.): principais perguntas do futuro empreendedor florestal. Rio Branco: Embrapa Acre, 2005. 88 p. (Embrapa Acre. Documentos, 97).

FIRMINO, A. C.; TOZZE JUNIOR, H. J.; FURTADO, E. L. First report of Ceratocystis fimbriata causing wilt in Tectona grandis in Brazil. New Desease Reports, v. 25, p. 24, 2012. DOI: 10.5197/ j.2044-0588.2012.025.024

FLORÉZ, J. B. Caracterização tecnológica da madeira jovem de teca (Tectona grandis L. f.). 2012. 85 f. Dissertação (Mestrado em Ciências Florestais) - Universidade Federal de Lavras, Lavras.

FONSECA, S. G. C.; BRAGA, R. M. C.; SANTANA, D. P. Lapachol: química farmacologia e métodos de dosagem. Revista Brasileira de Farmacognosia, João Pessoa, v. 84, n. 1, p. 9-16, 2003.

FRAGA, D. G.; SILVA JUNIOR, F. G.; GONÇALEZ, J. C. Avaliação da polpação soda de Pinnus taeda com adição de antraquinona. In: CONGRESSO IBEROAMERICANO DE INVESTIGACIÓN EM CELULOSA Y PAPEL: CIADICYP, 2., 2002, Campinas. [Anais]. [S.1: s.n.], 2002. 9 p.
GARCÍA, J. A. V.; DAOUD, R. Efeitos dos antioxidantes fenólicos na prática desportiva. Fitness \& Performance Journal, v. 1, n. 4, p. 21-27. 2002.

GIBAJA, S. Pigmentos naturales quinónicos. Lima: UNMSM, 1998. 277 p.

GOERGEN, L. C. G.; SOARES, J. C. W.; BERRA, E. F.; BRANDELERO, C.; PEREIRA, R. S. Índices espectrais para avaliação da dinâmica sazonal de clorofilas em folhas de Eucalyptus grandis. Pesquisa Florestal Brasileira, Colombo, v. 35, n. 82, p. 55-61, 2015. DOI: http//dx.doi.org/10.4336/2015.pfb.35.82.841

GONZÁLEZ,A. G.; BARROSO, J. T.; CARDONA, R. J.; MEDINA, J. M.; RODRIGUEZ LUIS, F. Distribuición de antraquinonas y compostos naftalénicos relacionados en Rubiáceas, Bignoniaceas e Verbanáceas. In: ANALES DE LA UNIVERSIDAD DE DE LA LAGUNA, 9., 1971, Tenerife. Anais... Laguna: ULLI, IC, 1971. p. 4-22.

GUERRA, S. C. S. Influência do teor de extrativos na resistência natural de cinco madeiras a cupins xilófagos. $2010.36 \mathrm{f}$. Monografia (Graduação em Engenharia Florestal) - Universidade Federal do Espírito Santo, Jerônimo Monteiro.

KLOCK, U.; MUÑIZ, G. I. B.; HERNANDEZ, J. A.; ANDRADE, A. S. Química da madeira. 3. ed. Curitiba: UFPR, 2005. 25 p.

MARTÍ, M. A. C. Aspectos ecotoxicológicos de las maderas tropicales. Observatório Medioambiente, n. 1, p. 257-267, 1998.

MENDOZA, Z. M. S. H.; BORGES, P. H. M. Estudo Fitoquímico da Folha de Teca. In: CONGRESSO BRASILEIRO DE CIÊNCIA E TECNOLOGIA, 1., e SIMPÓSIO DE CIÊNCIA E TECNOLOGIA DA MADEIRA DO RIO DE JANEIRO, 3., 2013, Petrópolis . Anais... Rio de Janeiro: UFRRJ, 2013. p. 98-101.

MOREIRA, R. Y. O.; ARRUDA, M. S. P.; ARRUDA, A. C.; SANTOS, L. S.; MÜLLER, A. H.; GUILHON, G. M. S. P.; SANTOS, A. S.; TEREZO, C. Antraquinonas e naftoquinonas do caule de um espécime de reflorestamento de Tectona grandis (Verbenaceae). Brazilian Journal of Pharmacognosy, v. 16, n. 3, p. 392-396, 2006.

MORILLO, P. Teñido de lana aplicando los colorantes de la hoja de Teca (Tectona grandis). 2009. $138 \mathrm{f}$. Tese (Doctorado en Ingenieria Quimica) - Escola Politécnica Nacional, Facultad de Ingenieria Quimica y Agroindustria, Quito.

MOTTA, J. P.; OLIVEIRA, J. T. S.; PAES, J. B.; ALVES, R. C.; DAMBROZ, G. B. V. Resistência natural da madeira de Tectona grandis em ensaio de laboratório. Ciência Rural, Santa Maria, RS, v. 43, n. 8, p. 1393-1398, 2013. DOI: 10.1590/S010384782013005000097

NIDAVANI, R. B.; MAHALAKSHMI, A. M. Teak (Tectona Grandis Linn.): renowned timber plant with potential medicinal values. International Journal of Pharmacy and Pharmaceutical Sciences, Academic Sciences, India, v. 6, n.1, p. 48-54, 2014.

OLIVEIRA JUNIOR, H. F.; ROSA, G. S.; SANTOS, A. B. Análise in vitro do efeito do extrato hidroalcoólico de Tectona grandis Linn. (Verbenaceae) obtido diferentes condições de termotratamento e in natura sobre o fungo Agrocybes perfecta (Rick) Singer. In: ENCONTRO REGIONAL DE INICIAÇÃO CIENTÍFICA, 1., 2012, Jaguariaiva. Anais... Jaguariaiva: [s.n.], 2012, p. 1-2. 
OLIVEIRA, J. T.; TOMASELLO, M.; SILVA, J. C. Resistência natural da madeira de sete espécies de eucalipto ao apodrecimento. Revista Árvore, Viçosa, MG, v. 29, n. 6, p. 993-998, 2005.

PAES, J. B.; MELO, J. J; LIMA, C. R. Resistência natural de sete madeiras a fungos e cupins xilófagos em condições de laboratório. Red de Revistas Científicas de América Latina, El Caribe, Espanha y Portugal. Cerne, Lavras, v. 13, n. 2, p. 160-169, 2007.

PINTO, M. L. Propriedades e características da madeira de teca (Tectona grandis) em função da idade. 2007. $124 \mathrm{f}$. Tese (Doutorado em Engenharia Civil) - Universidade Federal de Santa Catarina, Florianópolis.

POLTRONIERI, L. S.; ALFENAS, R. F.; ALFENAS, A. C.; VERZIGNASSI, J. R.; BENCHIMOL, R. L.; POLTRONIERI, T. P. S. Primeiro registro de Cylindrocladium parasiticum em plantas de teca no Pará. Summa Phytopathologica, Botucatu, v. 37, n. 3, p. 152, 2011. DOI: 10.1590/S0100-54052011000300014

SARTO, C.; SANSIGOLO, C. A. Cinética da remoção dos extrativos da madeira de Eucalyptus grandis durante polpação Kraft. Acta Scientiarum Technology, Maringá, v. 32, n. 3, p. 227-235, 2010. DOI: $10.4025 /$ actascitechnol.v32i3.4237
SILVA, M. N.; FERREIRA, V. F.; SOUZA, M. C. B. V. Um panorama atual da química e da farmacologia de naftoquinonas, com ênfase na $\beta$-Lapachona e derivados. Química Nova, Niterói, v. 26, n. 3, p. 407-416, 2003. DOI: 10.1590/S0100-40422003000300019

SOUSA, E. T. Quinonas no ar atmosférico: determinação, concentrações e correlações entre as fases vapor e particulada. 2012. 117 f. Tese (Doutorado em Química) - Instituto de Química, Universidade Federal da Bahia. Salvador.

SOUSA, O. V.; OLIVEIRA, M. S.; CUNHA, R. O.; COSTA, B. L. S.; ZANCANELLA, C. R.; LEITE, M. N. Avaliação da qualidade de matérias-primas de ruibarbo utilizadas em formulações farmacêuticas. Revista Brasileira de Farmacognosia, São Paulo, v. 13, p. 30-34, 2003.

SOUZA, R. F.; GUILHON, G. M. S. P.; AGUIAR, O. J. R.; SANTOS, A. S. Concentrados de metabólitos obtidos das folhas da teca (Tectona grandis (L.f)) para uso como corante. In: ENCONTRO DE PROFISSIONAIS DA QUÍMICA DA AMAZÔNIA, 10., 2007, Belém. Anais... Belém, UFPA, 2007. p. 1-5.

TSUKAMOTO FILHO, A. A.; SILVA, M. L.; COUTO, L.; MÜLLER, M. D. Análise econômica de um plantio de teca submetido a desbastes. Revista Árvore, Viçosa, MG, v. 27, n. 4, p. 487-494, 2003. 
